\title{
Mycological rationality: Heuristics, perception and decision-making in mushroom foraging
}

\author{
Roope O. Kaaronen*
}

\begin{abstract}
How do mushroom foragers make safe and efficient decisions under uncertainty, or deal with the genuine risks of misidentification and poisoning? This article is an inquiry into ecological rationality, heuristics, perception, and decision-making in mushroom foraging. By surveying 894 Finnish mushroom foragers, this article illustrates how socially learned rules of thumb and heuristics are used in mushroom foraging, and how simple heuristics are often complemented by more complex and intuitive decision-making. The results illustrate how traditional foraging cultures have evolved precautionary heuristics to deal with uncertainties and poisonous species, and how foragers develop selective attention through experience. The study invites us to consider whether other human foraging cultures might use heuristics similarly, how and why such traditions have culturally evolved, and whether early hunter-gatherers might have used simple heuristics to deal with uncertainty.
\end{abstract}

Keywords: bounded rationality, ecological rationality, expertise, foraging, heuristics, mushroom hunting

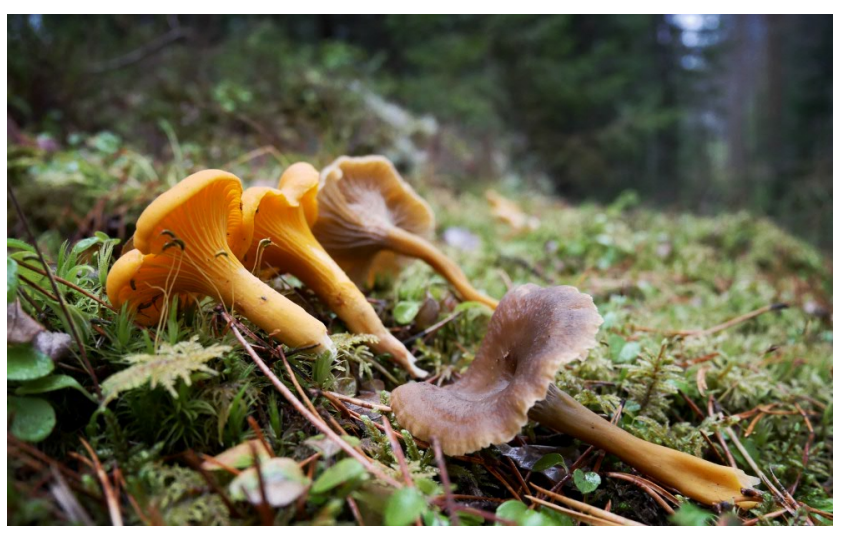

FIGURE 1: Uprooted chanterelles (Cantharellus cibarius, left) and funnel chanterelles (Craterellus tubaeformis, right) in their natural terrain. Photograph by author.

Mycology (noun): the scientific study of fungi. (Cambridge dictionary, 2019).

Note: The heuristics and rules discussed in this text should NOT be used as a guide for identifying mushrooms. Always consult a local expert and multiple information sources before picking or eating wild mushrooms.

I thank Jukke Kaaronen, Janne I. Hukkinen, Liam P. Satchell, Max Ritts, Peter M. Todd, Valentina Kieseppä, and members of the Environmental Policy Research Seminar for comments during earlier stages of this research. I also thank Ulrich Hoffrage, one anonymous reviewer, and editor Jonathan Baron for their helpful suggestions.

Copyright: (c) 2020. The authors license this article under the terms of the Creative Commons Attribution 3.0 License.

${ }^{*}$ University of Helsinki, Faculty of Social Sciences, Helsinki Institute of Sustainability Science, Unioninkatu 37, FI-00014 Helsinki. Email: roope.kaaronen@helsinki.fi. ORCID: 0000-0002-2414-6974.
All mushrooms are edible - but some only once. (Proverb).

\section{Introduction}

Imagine a forager in the wild. The hazy and damp forest is cluttered with a range of autumnal colors, fallen leaves and rotting foliage (Figure 1). The forager is confronted with dozens, or even hundreds, of barely identifiable or visible mushrooms, only a fraction of which are suitable for human consumption. Here, a single misidentification can lead to organ failure or death. Yet still, the forager is accompanied by children and adolescents who fare surprisingly well at the task, and the foragers are carrying hefty baskets filled with seasonal delicacies (Figure 2). How do they succeed in this?

One could assume the answer to be something as follows: "When encountering a mushroom in the wild, foragers use complex sets of rules and cognitive procedures to aid identification, and systematically weigh the risks or costs of poisoning against the benefits of good catch." However, recent research in decision-making invite us to consider another possibility: the use of simple rules of thumb or "fast and frugal" heuristics (Eisenhardt \& Sull, 2001; Gigerenzer \& Todd, 1999; Marewski et al., 2010; Sull \& Eisenhardt, 2015; Todd \& Gigerenzer, 2012). The general message from this area of research suggests that good judgments do not necessarily require complex cognitive processing, and that, particularly in environments with high degrees of uncertainty, people often resort to simple heuristics when making decisions. Moreover, in such environments, simple rules might systematically outperform more complex judgments (Kozyreva \& 


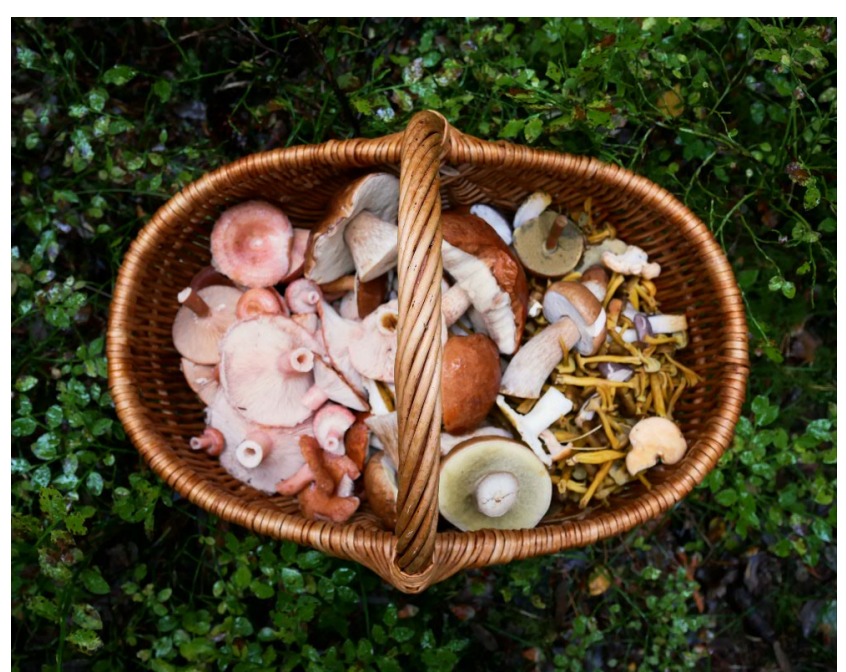

FIGURE 2: A typical foraging basket with an assortment of mushrooms. Photograph by author.

Hertwig, 2019; Marewski et al., 2010; Todd \& Gigerenzer, 2012).

This research article studies decision-making in Finnish mushroom foragers. Although mobility patterns and spatial abilities in mushroom foragers have been studied (PachecoCobos et al., 2010, 2019), the processes of decision making in foraging have received little attention (although see Bullock and Todd, 1999 for a simulation study). In the present study, 894 mushroom foragers, with a total of 22,304 years of foraging experience, are surveyed about their foraging rules, strategies, and habits. I argue that the theory of ecological rationality can help us understand the practice and success of mushroom foragers, but also discuss limitations of the framework in understanding the often-complex identification processes, where heuristics are complemented by complex or intuitive decision-making processes. The research questions of the present manuscript are:

1. How do mushroom foragers make decisions?

2. What, if any, (fast and frugal) heuristics or simple rules do mushroom foragers use when identifying mushrooms or making judgments about edibility?

Furthermore, this article explores how foragers leverage recurrent and reliable perceptual cues in their environment when searching for mushrooms, how foragers develop selective attention, and how culturally acquired knowledge is central to these processes. The results thus also contribute to the understanding of the cultural evolution of foraging practices and preserves traditional knowledge into scientific literature. Next to quantitative analysis, a variety of qualitative approaches are used to study foraging strategies and heuristics. This is a response to Herbert Simon's (2000, pp. 35-36) call for further focus on verbal protocols, written records and natural language when studying decision-making processes.
This article accompanies a previous autoethnographical and phenomenological study (Kaaronen, 2019). The present text contributes to taking research on ecological rationality "into the wild," assessing how well simple cognitive rules can be used to make decisions in noisy and uncertain realworld contexts. The results also invite the reader to consider how the verbally transmitted art of mushroom foraging has evolved culturally through social learning, and how earlier hunter-gatherer societies may have employed similar cognitive strategies to survive in high-uncertainty environments.

The results are mainly interpreted through the lens of ecological rationality. Broadly defined, research in ecological rationality emphasizes that the success of a decision depends on the context in which it is made, and that under particular environmental regularities even simple strategies or heuristics can be well-adapted and successful. Ecological rationality can therefore be understood in terms of cognitive success in the world, or the fit between the mind and the environment (Gigerenzer \& Todd, 1999; Kozyreva \& Hertwig, 2019; Todd \& Brighton, 2016).

Research in ecological rationality has worked to uncover the often successful, domain-specific simple heuristics which cognitively bounded humans employ particularly when faced with uncertainty in decision-making (Kozyreva \& Hertwig, 2019; Todd \& Gigerenzer, 2007, p. 167). It is thus the task of ecological rationality to uncover the "adaptive toolbox" of the mind (Todd \& Gigerenzer, 2012). The tools in this toolbox are heuristics, which are tuned to specific environmental regularities and designed for task-specific problems (Marewski et al., 2010, p. 106; Todd \& Brighton, 2016).

However, as is discussed below, not all of decision-making in mushroom foraging can be easily accommodated within the scope of ecological rationality. For one, the use of "fast and frugal" or simple heuristics in foraging is often accompanied by more complex, slow and intuitive decision-making. Second, whereas the research tradition in fast and frugal heuristics typically studies well-defined algorithmic search, stopping and decision rules in decision-making, many of the simple heuristics used by foragers are fuzzier, rather resembling rough "guidelines, principles or routines, tailored to specific situations or domains" (Marewski \& Hoffrage, in press). These are more akin to "simple rules" as defined by Eisenhardt and Sull $(2001 ; 2015)$. Such heuristic principles have been discussed to be particularly suitable for complex and uncertain environments (Marewski \& Hoffrage, in press).

The present paper studies decision-making in ecological context and therefore, before moving on, it is particularly important to define the context of mushroom foraging in Finland, where mushroom foraging is considered traditional cultural heritage. The practice of mushroom foraging has been transmitted, mainly verbally, from one generation to the next, and even today most foragers learn the practice from their family or relatives. The precise origins of mush- 

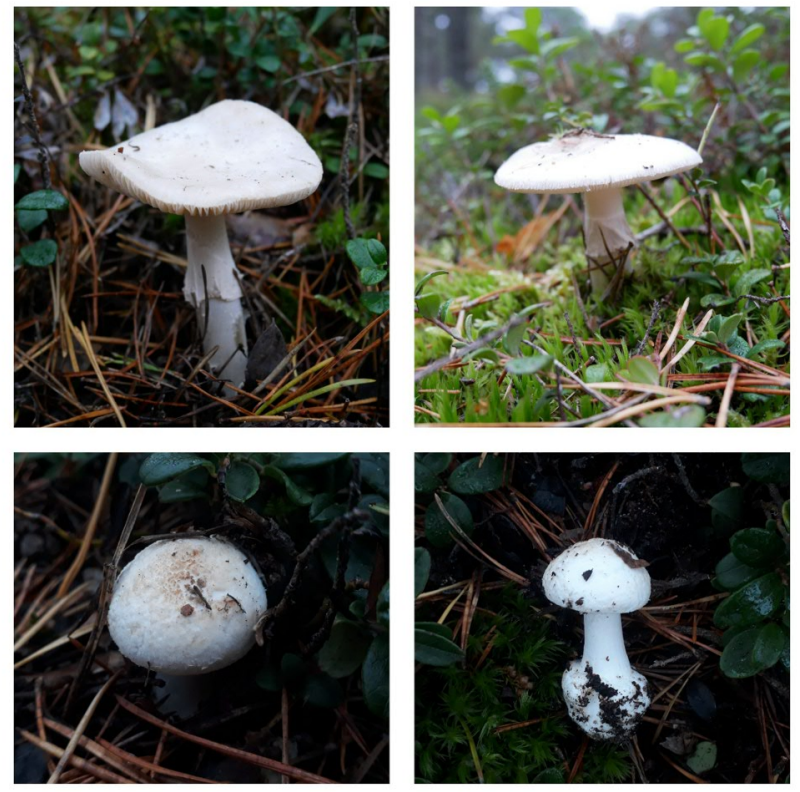

FIgURE 3: A collage of fruiting bodies of the deadly poisonous Amanita virosa, commonly known as the destroying angel. Pictured are two grown mushrooms (above) and two photos of a young mushroom (below). Notice how similar particularly a young $A$. virosa (bottom left) can look to a typical champignon. Bottom right illustrates an exhumed young $A$. virosa, with an onion-like bulb at the base, typical to the species. A. virosa can also be recognized by the hanging ring on its stem (seen in the two pictures on top; it has not yet developed in the lower pictures) and its completely white color. $A$. virosa is one of the most poisonous mushrooms in the world-one cap is enough to kill an adult human. It grows abundantly in Finland. Photographs by author.

room foraging in Finland are unknown, but it is believed to have been influenced by varying foraging cultures from Russia and Sweden. These cultural influences also varied in their preferences: influence from Russia brought in the use of milk-caps (Lactarius) and other mushrooms that are typically pickled, whereas Swedish influence is seen in the high valuation of mushrooms such as porcinis and chanterelles. The Finnish foraging tradition is typically considered a mixture of these two cultures. The popularity of foraging in Finland is at least partly due to public education, which has taught the people to survive in times of food shortage. Mushrooms are mainly foraged for food, although they are also used for other purposes, such as dyeing. Mushroom foraging is also considered by many a recreational activity (Elävä perintö, 2019).

Some 5400 fungal species grow in Finland, about 2000 of which grow spore-bearing fruiting above-ground bodies called mushrooms (Elävä perintö, 2019). Of the mushrooms in Finland, at least a few hundred are suitable for regular human consumption (Korhonen, 2015). The annual growth of mushrooms in Finland ranges from 1.5 to 4 billion kilograms, of which humans harvest 2 to 10 million kilograms. Mushrooming is a highly popular activity, and an estimated over $40 \%$ of adult Finns go mushroom hunting on a yearly basis (Metsäntutkimuslaitos, 2010). Mushrooming is considered an "everyman's right", which entitles "everyone in Finland to enjoy outdoor pursuits regardless of who owns or occupies the area" (Ministry of the Environment, 2019).

Although mushroom foraging is mainly taught verbally and in practice, mushroom identification books are also popular. The first known Finnish mushroom identification book was published in 1863 (Hisinger, 1863). Since then, mushroom books have gained wide popularity, and are today a staple in Finnish households and summer cottages. Mushroom identification books include detailed instructions for safe identification of mushrooms. Such instructions range from taxonomical features to perceptual cues, which include descriptions of a wide range of visual, olfactory, haptic and gustatory cues for mushroom identification, as well as some simple rules of thumb for safe foraging (Korhonen, 2015).

Among the Finnish fungi grow dozens of poisonous species, of which at least six are deadly. Many of the deadly species grow abundantly. Whilst mushroom related fatalities and serious accidents do occur every now and then, they are rare. Between 1969 and 2017, a total of nine people died of mushroom poisoning in Finland, and five people received a mushroom poisoning related liver transplant (Maaseudun tulevaisuus, 2017). Accidents are generally attributed to misidentification, where a poisonous mushroom is confused for an edible one. For instance, a deadly Amanita virosa (Figure 3) might resemble a highly valued mushroom of the Agaricus genus (including the cultivated portobello and champignon mushrooms found in supermarkets worldwide).

Mushroom foraging is an activity characterized by considerable uncertainty. Mushroom development is highly variant. Local populations or individuals might exhibit unusual color, shape, or size for the species, making identification difficult on the spot. It is easy to confuse an edible mushroom for a poisonous one, and distinguishing between similar species can be near impossible without the help of microscopy. Different conditions in humidity, moisture, sunlight, weather or soil quality can have a considerable effect on the appearance of mushrooms, and mushrooms can grow in unexpected patches or environments. Young mushrooms also generally differ greatly in their features from fully grown ones (some telltale cues may yet be undeveloped), making identification difficult and increasing the risk of mistaking a poisonous species for an edible one (see, e.g., Figure 3). (Korhonen, 2015.)

This article is structured as follows. First, Study 1 is presented and its results are discussed. Study 1 uses a variety of qualitative and quantitative methods to uncover the heuristics and other decision-making and search strategies used 
TABLE 1: Gender distribution of respondents.

\begin{tabular}{lll}
\hline Female & 757 & $84.7 \%$ \\
Male & 124 & $13.9 \%$ \\
Other or do not wish to tell & 13 & $1.5 \%$ \\
Total & 894 & $100 \%$ \\
\hline
\end{tabular}

by foragers. Second, Study 2 is presented and its results are discussed. Study 2 involves a search task, studying the use of visual cues in foraging. The manuscript ends with General Discussion.

Data and $\mathrm{R}$ code are available at https://github.com/ roopekaaronen/mushroom/

\section{Study 1}

Using an online survey design, Study 1 charts out the heuristics, simple rules and strategies that foragers report using when mushroom hunting, along with other general foragingrelated questions and demographics. A link to an online survey was shared on Finnish mushroom foraging societies on their social media platforms. These groups are relatively popular and active: the largest group has over 30 000 members. 894 unique responses were registered during April-June 2019. Participants were recruited with informed consent. The survey was conducted in Finnish. All questions and some qualitative responses in this manuscript have been translated from Finnish to English by the author.

\subsection{Method}

\subsubsection{Participants}

The study was completed by 894 Finnish or Finnish-speaking mushroom foragers. Table 1 illustrates the gender distribution of the respondents. $84.7 \%$ of respondents were female. This gender ratio is partly a product of the higher representation of females in the population of mushroom foragers. According to Finnish statistics in 2010, 44.7\% of Finnish females foraged for mushrooms on a yearly basis, whilst the share was 35.9\% for men (Metsäntutkimuslaitos, 2010).

Respondents were asked where they learned to forage from (Table 2). The most common response $(n=609,68.1 \%)$ was from their parents. Mushroom foraging is still largely transmitted through vertical cultural transmission (from parents and grandparents), although expert information sources such as foraging guides $(\mathrm{n}=576,64.4 \%)$ are almost as popular. Note that the share of learning from the internet is particularly likely to be biased here, since the participants were recruited from online groups. However, the results do reflect a shift in social learning strategies, as new information sources such as online internet groups become
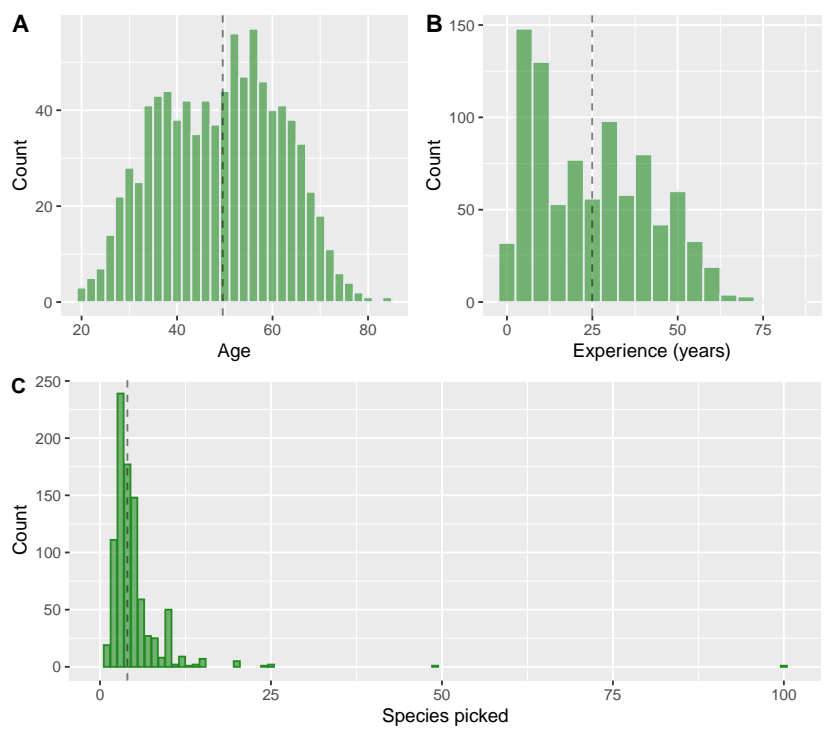

Figure 4: Descriptive statistics. A: Age distribution with dashed line at mean age of foragers. B: Experience distribution with mean experience (years) of foragers. C: Figure 3. Distribution of number of species picked on one foraging trip with median.

increasingly popular in spreading traditions that were previously mainly oral. These results also align with general findings that oral traditions are increasingly complemented by co-existing scholarly, expert and online sources (Mazzocchi, 2006; Cocq, 2013).

The respondents' motive for foraging was also surveyed (Table 3). Interestingly, the most common answer was "for fun or hobby" ( $n=777,86.9 \%)$. "For food" was the second most common with $770(86.1 \%)$ mentions (recall that mushrooms are also picked for dyeing, beverages and other uses). The results suggest that foraging is considered much more than food collection, and is an important form of leisure activity, nature connection, and social life.

The mean age of the respondents was 49.6 years (Figure 4A), which is considerably higher than the mean age of Finns, 42.3 years (Tilastokeskus, 2019). The foragers surveyed were highly experienced (Figure 4B), with a mean experience of 25 years of foraging (although note the peak in foragers with under 10 years of experience). The survey reached a total of 22,304 years of mushroom foraging experience. Foragers were also asked how many species of mushrooms they forage on their average trip (Figure 4C). The distribution is highly right-skewed, with most foragers focusing selectively on under 5 species at a time. Some foragers, however, reported up to 49 or even 100 species (including one forager with 60 years of experience). ${ }^{1}$

\footnotetext{
${ }^{1}$ Whilst these are technically feasible, particularly since foraging societies include experts such as mycologists, there is also a chance that the higher-end answers mistook "species" for individual mushrooms, or that of a typo.
} 
TABLE 2: Who or where did you learn to forage from? (Select all that apply.)

\begin{tabular}{ccccccc}
\hline Parents & Grandparents & Other relatives & Books & Internet & Course & Other* \\
609 & 182 & 149 & 576 & 311 & 142 & 209 \\
$68.1 \%$ & $20.4 \%$ & $16.7 \%$ & $64.4 \%$ & $34.8 \%$ & $15.9 \%$ & $23.4 \%$ \\
\hline
\end{tabular}

*Answers included friends, spouses, colleagues, school, among others.

TABLE 3: Why do you forage? (Select all that apply.)

\begin{tabular}{lcccccc} 
Food & Fun/hobby & Research or science & Relax & Nature experience & Exercise & Social activity \\
770 & 777 & 49 & 707 & 726 & 514 & 288 \\
$86.1 \%$ & $86.9 \%$ & $5.5 \%$ & $79.1 \%$ & $81.2 \%$ & $57.5 \%$ & $32.2 \%$ \\
\hline
\end{tabular}

\subsubsection{Design}

Mushroom foraging heuristics were surveyed by asking the following open questions:

1. What rules of thumb do you use pertaining to safe foraging?

2. What rules of thumb do you use pertaining to the identification of edible or poisonous mushrooms?

3. What rules of thumb do you use pertaining to identifying good foraging patches or finding mushrooms? ("Rule of thumb" [Finnish: "nyrkkisääntö", literally "fist-rule"] is a well-known colloquialism in the Finnish language and is synonymous to a simple rule or heuristic.)

Foragers were also presented with eight statements to survey their mushroom hunting experience and strategies on a five-point scale. The statements were (with names used hereafter):

Hunch When I go mushroom foraging, I have a strong hunch of which mushrooms I expect to find.

Heuristics I often make use of rules of thumb when I forage.

Glance With a quick glance of a given terrain, I know which mushrooms could grow in the area.

Apaja I keep the knowledge of bountiful mushroom hunting patches ("apaja") to myself or my close ones. (Translation note: The Finnish word "apaja" does not directly translate into English. In this context, it means an area that is known or expected to reliably and recurrently grow (specific species/genera of) mushrooms.)

Experience I consider myself an experienced mushroom forager.

Familiar I mainly forage in familiar terrain.

Protect I would rather protect a forest where I forage than other forests which I visit.

Delicious If I search for a specific delicious or precious species of mushroom, I don't pick other species.

\subsubsection{Procedure}

For the three open questions, respondents were encouraged to answer with as many rules of thumb as they could recall. Responses were analyzed using inductive content analysis (Elo \& Kyngäs, 2008). This involved an initial process of identifying recurrent themes or patterns in the qualitative data, after which the themes were classified and coded systematically. Emergent themes and patterns (recurring rules of thumb, heuristics and other foraging strategies) were coded and their frequencies analyzed. Note that since the questions were open ${ }^{2}$, the true use of the mentioned heuristics is likely to be more common than the number of their mentions in the dataset (respondents might not have remembered that they use a particular heuristic at the time of response, or were otherwise unable to explicate a rule they use).

\subsection{Results}

First, as background information, the responses to the eight general foraging questions are analyzed. Results are shown in diverging stacked bar charts in Figure 5. Notably, 77\% of foragers report that they often use rules of thumb when foraging.

An exploratory correlation plot (with Pearson correlation coefficients, Figure 6) was produced from the eight questions above as well as the variables age (Age), foraging experience in years (Experience_years), and average number of species picked (Species). Acknowledging that an exploratory correlation plot should be interpreted with caution, some interesting relations seem to exist particularly between the experience variables (Experience and Experience_years) and having hunches (Hunch: $r=0.32$ and 0.21 , respectively) and anticipations (Glance: $r=0.49$ and 0.33 ) of what mushrooms one expects to find. Perhaps most interesting for the context of the present article, however, is that the use of heuristics

\footnotetext{
${ }^{2}$ Open questions were preferred since there exists little previous literature on the subject to inform sufficiently detailed closed questions.
} 


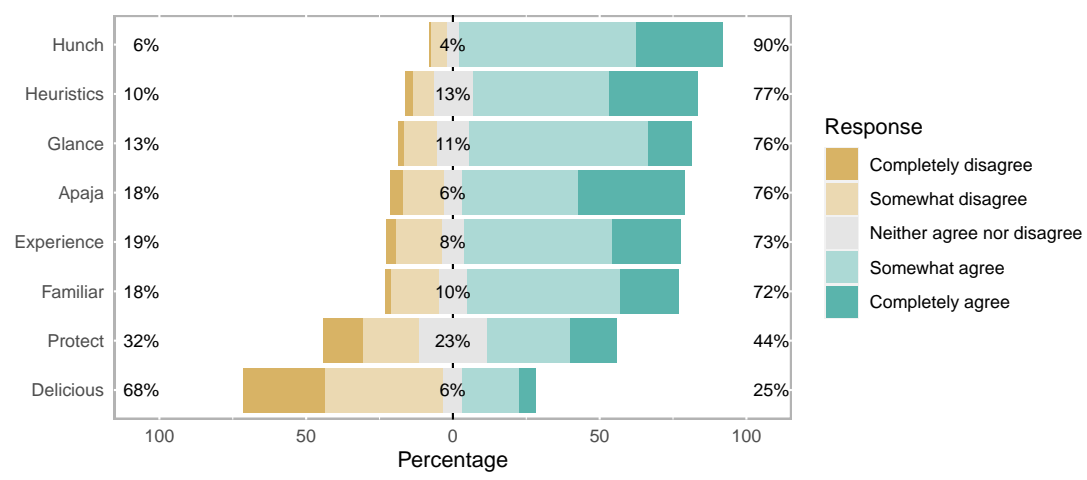

FIGURE 5: Diverging stacked bar charts of the eight general foraging questions asked. The table illustrates percentages of respondents who agree with (right), disagree with (left), and are neutral (center) regarding the statement.

(Heuristics) does not appear to be correlated with the experience variables $(\mathrm{r}=0.03$ and -0.03$)$. That is, heuristics are used by experts and novices alike.

Second, the results of the three open foraging questions are analyzed. The reported heuristics or strategies are defined in Tables 4-6 with frequencies of their mentions by respondents, along with representative examples from the qualitative dataset.

A total of 22 recurrent rules or strategies for safe foraging were identified (Table 4). Overall, foragers had little trouble with this question. The heuristic "Only pick mushrooms you can identify (with certainty)" was by far the most common with 525 instances.

A total of 19 rules for identifying edible or poisonous mushrooms were identified (Table 5). Note that the answers have some overlap with the heuristics reported in Table 4. A particularly common answer with 229 instances was identifying an edible mushroom, or specifically an edible milkcap (Lactarius), by the latex ("milk") the mushroom exudes when cut (see Figure 7). Also notably, the heuristic "Completely avoid or be extra cautious with white mushrooms (due to similarity with Amanita virosa)" was mentioned 112 times.

Verbally defining a good foraging patch (Table 6) proved to be a trickier task. 17 rules were identified. The most common instances were search heuristics related to finding some of the most valued mushrooms (funnel chanterelles, chanterelles, Gyromitra esculenta, and boletes), and identifying associations between fungi and plants, such as "chanterelles grow near birch trees" (108 instances) or "funnel chanterelles grow near spruces" (49 instances).

Next, the results are analyzed in terms of a typology of decision-making in mushroom foraging. ${ }^{3}$ These include four distinct (but often complementary) processes by which the results suggest that mushroom identification or search happens (in no particular order): 1., Heuristics for identifying

\footnotetext{
${ }^{3}$ I thank Jonathan Baron for suggesting this typology for organizing the
} results. mushrooms; 2., Meta-heuristics; 3., Intuitive pattern recognition; and, 4., Full identification. The foraging strategies or heuristics in Tables 4-6 include a column which designates which of these four categories the strategy/heuristic most resembles.

1. Heuristics for identifying mushrooms involve the identification of particular cues, which are usually named, like the color, smell (Figure 7), or unique feature of a mushroom. These are most similar to those rules typically discussed in the context of fast and frugal heuristics, i.e., using a single cue or a limited number of cues to discriminate between alternatives. These heuristics involve the use of simple rules to either identify a mushroom (as poisonous or edible) or the use of simple cues during search.

Some notable examples include using a single cue to reject subsets of mushrooms:

- Completely avoid or be very cautious with white mushrooms (due to similarity with Amanita virosa). (Tables 4 and 5.)

- Completely avoid or be extra cautious with mushrooms with spotted caps (many poisonous Amanita have spotted caps). (Table 5 and Figure 8.)

- Or using a simple cue to identify an edible mushroom species: Identify an edible mushroom, or specifically an edible milk-cap (Lactarius), by the latex ("milk") the mushroom exudes when cut. If it bleeds white "milk", the mushroom is judged edible (and if not, it might be poisonous). (Tables 4 and 5, Figure 7.)

- Or using a simple cue when searching for mushrooms (including the identification of symbiotic or mychorrizal ${ }^{4}$ associations between fungi and plants): Chanterelles (Cantharellus cibarius) grow near birch trees. (Table 6.)

\footnotetext{
${ }^{4}$ Encyclopaedia Britannica (2019) defines mychorrizal relationships as follows: "Mycorrhiza is a non-disease-producing association in which the fungus invades the root to absorb nutrients. Mycorrhizal fungi establish a mild form of parasitism that is mutualistic, meaning both the plant and the fungus benefit from the association. [...] By sharing the products it absorbs from the soil with its plant host, a fungus can keep its host alive."
} 
TABLE 4: What rules of thumb do you use pertaining to safe foraging? ( $N=894)$ Categories: $1=$ Heuristics for identifying mushrooms; 2 = Meta-heuristics; 3 = Intuitive pattern recognition; 4 = Full identification.

\begin{tabular}{l} 
Description of rule or heuristic \\
\hline Only pick mushrooms you can identify (with certainty). \\
Carry sufficient equipment, including: identification book, \\
gumboots, mushrooming knife, matchsticks, map, etc.
\end{tabular}

Identify an edible mushroom, or specifically an edible milk-cap (Lactarius), by the latex ("milk") the mushroom exudes when cut. If it bleeds white "milk", the mushroom is judged edible (and if not, it might be poisonous).

Only eat mushrooms you can identify with certainty.

Keep different species or genera of mushrooms in different containers ( since, e.g., some are non-edible or even deadly before blanching, some are picked for colouring, etc.).

Completely avoid or be very cautious with white mushrooms (due to similarity with Amanita virosa).

Carry unidentified or uncertain mushrooms in a different container.

When identifying a mushroom, consult multiple information sources, including other people, books and online communities.

Learn to identify new mushrooms one or a few species at a time (or per year).

Only pick mushrooms from clean environments/far from roads. Be aware of your starting point and/or surroundings, beware of getting lost.

Learn to identify (the most common) poisonous mushrooms an lookalikes.

Tell others where you are going/where you are.

Keep your mushrooming knife in its case or in the basket when foraging.

Forage in familiar terrain and areas.

Use other senses (smell, touch, taste when appropriate) to ensure successful identification.

Identify the mushroom in its natural terrain, and/or ensure it is not close to poisonous mushrooms.

Identify the mushroom once more at home, or when cleaning or preparing the mushroom.

Pick, identify and maintain whole mushrooms without breaking them (until preparation).

Do not forage alone.

Make sound (to scare off wildlife such as bears, wolves or snakes).

Boletes (Boletales spp.) are not poisonous.

\section{Examples}

Only pick those mushrooms that you can identify with certainty. Don't pick mushrooms that you can't recognise.

A first-aid kit is carried in the backpack. A water bottle to prevent dehydration. Phone charged with prepaid and an emergency application installed. Dress for the weather.

All milk-caps that excrete milky sap are edible.

I don't eat those mushrooms that I can't be $100 \%$ sure

I've identified.

I sort mushrooms by the species into paper bags as soon as I pick them into my basket.

I do not pick completely white mushrooms. Be extra careful with white mushrooms.

I carry a separate bag where I pick those mushrooms that I want to examine more.

I take uncertain mushrooms to be identified by an acquaintance. As a tool for identification I use the internet, books and hobbyist forums.

Learn one new species at a time. I learn one new species each year.

I don't pick mushrooms next to big roads.

I avoid being lost by observing my route. I get my direction from the sun. On cloudy weather I try to remember the form of the terrain.

Each spring I learn the most poisonous mushrooms in Finland.

I always tell my family where I am going mushroom foraging.

Always place the mushroom knife in the basket when you don't need it.

I forage in familiar terrain.

A mushroom should always be looked/smelled/felt for many identification cues. One is not enough to define a species.

Aim to identify mushrooms in the terrain, don't wait until you are home.

I go through the picked mushrooms once more when I unpack my catch.

I pick whole mushrooms, so they are easy to identify.

I don't go mushroom foraging alone, I have a bad sense of direction.

Make sound in the forest, so that animals give way.

1

.

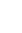


TABLE 5: What rules of thumb do you use pertaining to the identification of edible or poisonous mushrooms? ( $\mathrm{N}=894$.) Categories: 1 = Heuristics for identifying mushrooms; 2 = Meta-heuristics; 3 = Intuitive pattern recognition; 4 = Full identification.

\begin{tabular}{l} 
Description of rule or heuristic \\
\hline Identify an edible mushroom, or specifically an edible milk-cap \\
(Lactarius), by the latex ("milk") the mushroom exudes when \\
cut. If it bleeds white "milk", the mushroom is judged edible \\
(and if not, it might be poisonous). \\
Only pick mushrooms you can identify (with certainty).
\end{tabular}

Completely avoid or be extra cautious with white mushrooms (due to similarity with Amanita virosa).

When identifying a mushroom, consult multiple information sources, including other people, books and online communities.

Pay attention to the smell of the mushroom.

Boletes (Boletales spp.) can be identified by the spongy pores ("tubes") underneath their caps. (N.B. Related to heuristic below.)

Boletes (Boletales spp.) won't kill you (but not all are edible). (N.B. Some mildly poisonous boletes grow in certain southern regions of Finland but are rare overall.)

Learn to identify (the most common) poisonous mushrooms.

Learn to identify the (poisonous) lookalike species of edible mushrooms (and/or take caution when foraging mushrooms with lookalikes).

Avoid webcaps (Cortinarius) or do not pick them at all.

Amanita (which are often poisonous) generally have bulbs in their stems.

No heuristics suffice for identifying an edible mushroom (or distinguishing an edible from a poisonous one).

Pay attention to the terrain and natural environment when identifying a mushroom.

Only eat mushrooms you can identify with certainty.

Distinguish a porcini (Boletus edulis) from a lookalike but non-edible bitter bolete (Tylopilus felleus) by the netlike pattern ("fishnet stockings") on its stalk (bitter boletes have darker patterns).

Be cautious with mushrooms with a ring (skirt) around their stalk (many poisonous Amanita have this).

Completely avoid or be extra cautious with red mushrooms (due I do not pick any white or red mushrooms at all. to similarity with Amanita muscaria).

Completely avoid or be extra cautious with mushrooms with spotted caps (many poisonous Amanita have spotted caps).

Learn to identify new mushrooms one or a few species at a time I only learn one new mushroom species at a time. (or per year).
Examples

If a milk-cap excretes white milk, it is edible (an easy rule even for a child). If a milk-cap excretes colourless sap, it is poisonous.

I only pick those mushrooms that I recognise with

I do not pick any white mushrooms.

1121

If you don't recognise a mushroom you pick, utilise certainty.

The smell is an important part of mushroom

The curry milk-cap smells like curry.

Boletes have tubes. Boletes that look normal are usually edible, but I don't pick those boletes that I can't recognise.

Tubes (...) give the impression that it can't be a very poisonous species.

Boletes and russulas don't include poisonous species (in 40 easily be distinguished by tasting.

There aren't that many extremely poisonous mushrooms in Finland. Once you can identify those, you won't get yourself killed.

I remember the identification cues of those edible precision.

I don't pick any webcaps, even though they include edible ones.

Amanita have a bulb on their stem.

There are no rules of thumb to distinguish a poisonous mushroom from an edible one if they look a lot like each other.

I suppose this is a rule of thumb, too: There are no rules of thumb to pick mushrooms, you must know them.

Identify a mushroom in the environment it grows in.

I do not taste or eat unknown mushrooms.

$18 \quad 2$

A bad girl wears black stockings.

$17 \quad 1$

If it has a ring, I won't pick it.

No mushrooms with spots.

10

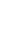


TABLE 6: What rules of thumb do you use pertaining to identifying good foraging patches or finding mushrooms? (N=894.) Categories: 1 = Heuristics for identifying mushrooms; 2 = Meta-heuristics; 3 = Intuitive pattern recognition; 4 = Full identification.

Description of rule or heuristic
Chanterelles (Cantharellus cibarius) grow near birch
trees.
Forage in familiar areas known to have good catch
(particularly since many species grow in the same spot
consecutive years).
Gyromitra esculenta (a false morel) grows in recently
logged forests.

Funnel chanterelles (Craterellus tubaeformis) grow in mossy terrain.

Funnel chanterelles grow near spruces.

A general preference towards old forests.

Mushrooms (in general) are best found in mossy terrain.

Funnel chanterelles can be found on sloped terrain.

Search for mushrooms near (forest) paths.

Boletes (particularly pine boletes, Boletus pinophilus) grow by pine trees.

A general preference towards sloped terrain.

Milk-caps (particularly rufous milk-caps) grow in dry heath forests.

If you find one mushroom (particularly funnel

chanterelles and chanterelles), you are likely to find more of the same in the immediate vicinity.

A general preference towards spruce forests.

Look for terrain that looks similar to previously proven foraging patches.

Trust in instinct (and not explicable rules of thumb) when foraging.

Identify a good foraging patch by its smell.

\section{Examples}

Count Cat.

A chanterelle yearns for a birch tree. Chanterelles $108 \quad 1$ thrive by birches.

Since a little child I have visited the same places. I $107 \quad 2$

can find what I'm searching for there.

Mushrooms have a habit of growing in the same places year after year.

I search for false morels in felled areas where the ground surface has been broken.

One should search for false morels in areas that were logged a couple of years ago.

A funnel chanterelle terrain can be recognised from a thick layer of moss.

Funnel chanterelles thrive in spruce forests.

Generally, the older the forest the more mushrooms.

A thick and moist terrain implies a good apaja. $\quad 41 \quad 2$

$\begin{array}{lll}\text { Funnel chanterelles thrive in sloped terrain. } & 39 & 1\end{array}$

Some good edible mushrooms seem to grow more $35 \quad 2$ near paths.

Boletes can be found in boreal pine forests.

$23 \quad 1$

Often slopes are good places.

$22 \quad 2$

One should search for rufous milk-caps in dry heath forests.

Once you see one funnel chanterelle, it is likely that you are standing on an abundance of them.

Old mossy spruce forests draw me towards them. $\quad 16 \quad 2$

At a new area, I aim to compare the terrain to places I am familiar with. This is how I deduce possible species I might encounter.

$21 \quad 2$

162

$13 \quad 3$
intuition, it often helps me to find boletes, chanterelles and especially funnel chanterelles.

A certain smell of mushrooms guides me to stop at $\quad 8 \quad 3$ the right places. I believe it is a mixture of moisture and something else. 


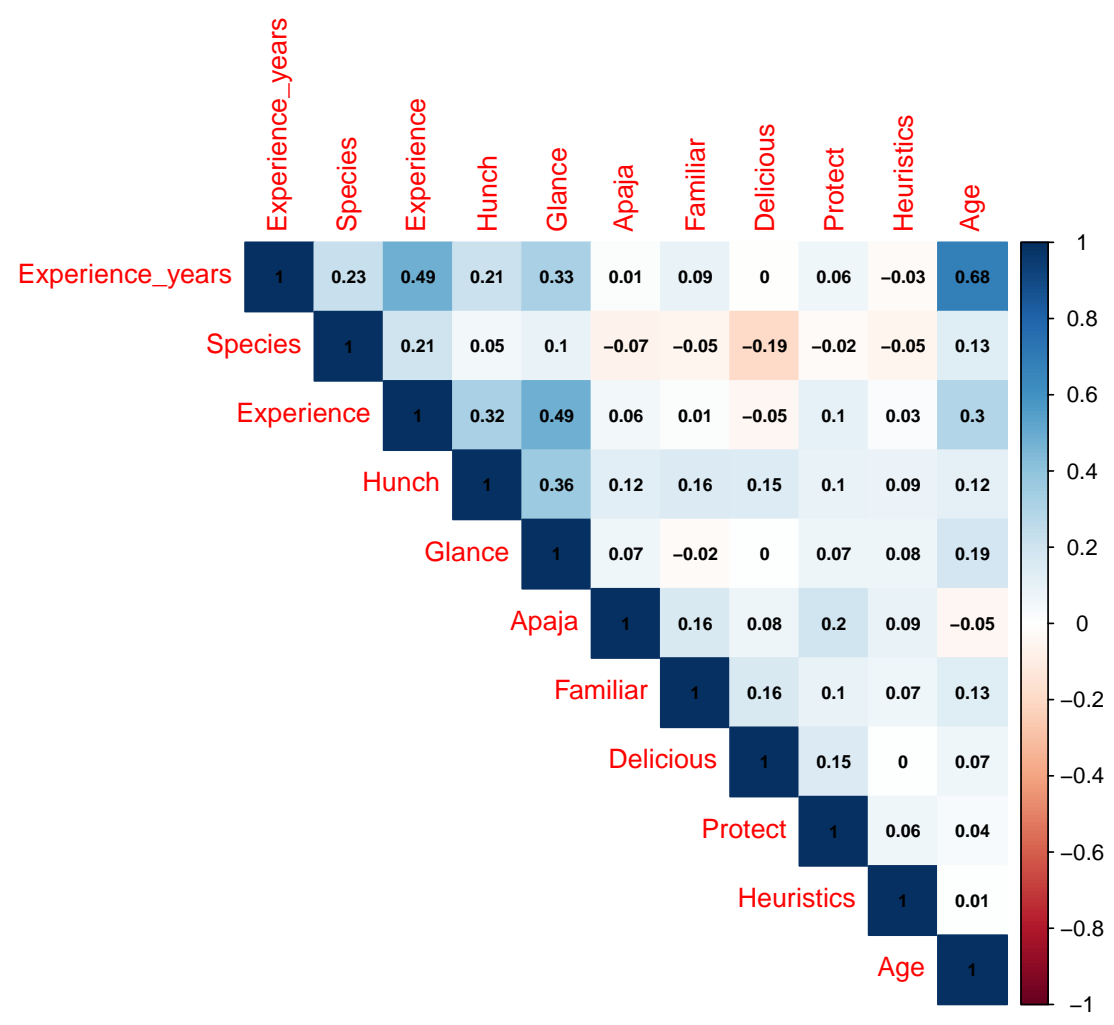

FIGURE 6: An exploratory correlation plot with Pearson correlation coefficients.

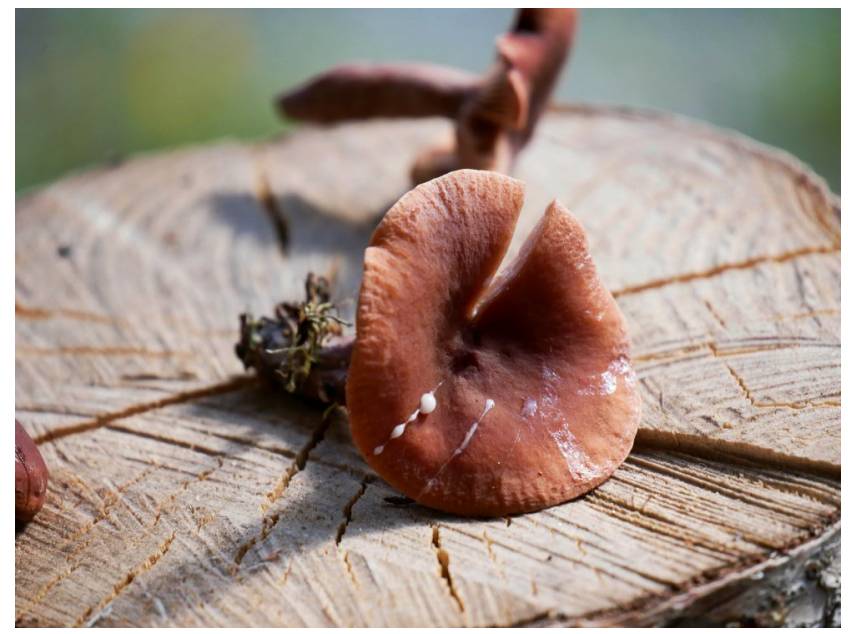

Figure 7: An exhumed curry milk-cap (Lactarius camphoratus), with cuts in its cap. Notice the white latex (or "milk") bleeding from the bottom-left of its cap, where it has been freshly cut with a mushroom knife. The curry milk-cap is also distinguishable by its distinct curry-like odor. Photograph by author.
- Funnel chanterelles (Craterellus tubaeformis) grow in mossy terrain. (Table 6, Figure 9.)

2. Meta-heuristics are heuristics or simple rules for organizing or conducting a foraging task. They are crude and generalizable guides for the search or decision-making procedures and are therefore distinguishable from the more specific mushroom identification heuristics. They are thus closer relatives to what Eisenhardt and Sull (2001; 2015) describe as "simple rules". More specifically, they include "how-to" rules (rules that guide successful behavior) and "boundary rules" (rules that define the boundaries within which the foraging task is to be practiced), or other heuristics that resemble rough guidelines or principles. Boundary rules particularly include precautionary heuristics, such as the general tendency to pick only those mushrooms one can identify with certainty,

- Only pick mushrooms you can identify (with certainty). (Tables 4 and 5.) This is due to the simple recognition of the fact that false positives (when making judgments of "edibility") are much worse than false negatives. There is therefore little tolerance of uncertainty in the practice (see Discussion below). 


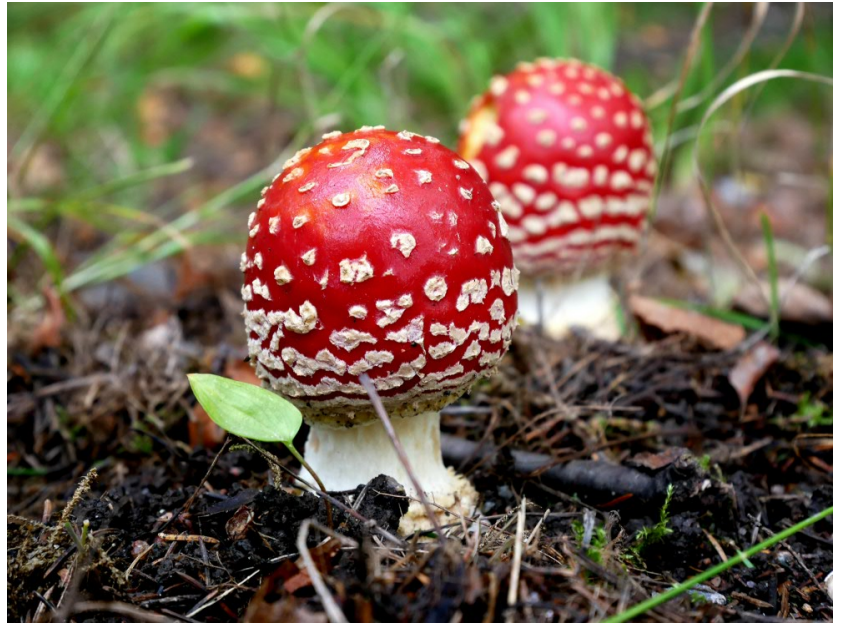

FIGURE 8: Some foragers report completely avoiding red and/or spotted mushrooms. This is generally due to similarity with the (in)famous poisonous fly agaric (Amanita muscaria) pictured above. Some foragers also mentioned they would not identify a fly agaric from the spots alone, since these might be washed away by rain, emphasizing that individual cues often do not suffice for safe identification. Notice also the bulb at the lower stem of the toadstool, and that the ring on the stem (typical to Amanita, see Figure 3) has not yet developed for these young individuals. Photograph by author.

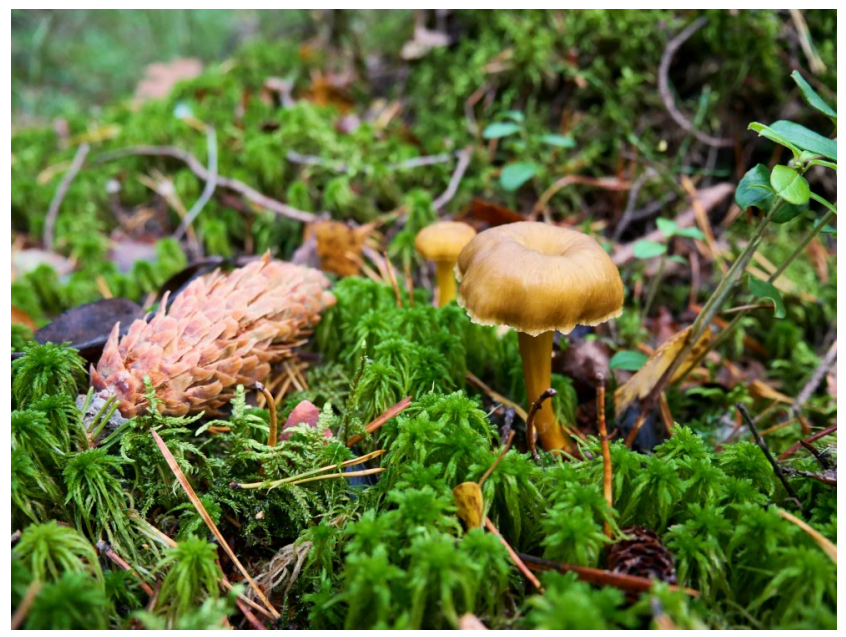

FIGURE 9: Three search heuristics in one picture: A funnel chanterelle thrives in mossy terrain, often grows near spruces (notice the spruce cone), and rarely grows alone (notice the smaller funnel chanterelle in the background). Photograph by author.

How-to rules involve, for example, strategies for setting an appropriate pace of learning:

- Learn to identify new mushrooms one or a few species at a time (or per year). (Table 4.)

- Or general rules for safe foraging, such as: Keep dif- ferent species or genera of mushrooms in different containers (since, e.g., some are non-edible or even deadly before blanching, some are picked for coloring, etc.). (Table 4.)

3. Intuitive pattern recognition. Although Study 1 was not specifically designed to study intuitive pattern recognition, many respondents report using intuition and pattern recognition when foraging, particularly during search for mushrooms. For instance, some foragers described their foraging practices as follows:

- "I can't put my finger on it, it is instinctive."

- "I am an instinctive forager. I do not identify consciously what factors are in place when I feel the intuition."

- "Mostly I recognize foraging patches intuitively, without rules of thumb. (...) I believe this owes to my experience of foraging every autumn with my grandparents when I was one to fifteen years old."

- "I don't know. My instinct drives me just like it drove my father back in the days. I haven't given it much thought, but I rarely miss the target."

- "It is difficult to describe with words. The general outlook [of the forest guides my foraging]. My instinct drives me to the right places."

- "Identifying a mushroom species is hard to describe in words. It is born from experience and practice. Next to looks and smell, it is affected by how the mushroom feels on your fingers and how it breaks when bent, how and where it grows, and the whole impression, which you recognize intuitively and near-instantly."

This intuitive pattern recognition might involve the use of exemplars (Jones et al., 2000; Karlsson et al., 2008). Here, foragers are able to identify categories (such as good foraging patches or edible mushroom species) effortlessly and without specific feature analysis, since during their years of foraging experience, they have acquired a large number of instances stored in memory (exemplars), which enable foragers to make unconscious similarity matches and category judgments between exemplars and newly encountered stimuli. Similar decision-making has been documented with, e.g., medical professionals (Norman et al., 2007; Norman \& Brooks, 1997). The use of intuition and exemplars is most obvious with the following reported strategies:

- Look for terrain that looks similar to previously proven foraging patches. (Table 6.)

- Trust in instinct (and not explicable rules of thumb) when foraging. (Table 6.)

4. Full identification of the mushroom, which includes access to the knowledge or memory of what species/genus a mushroom belongs to and whether the mushroom is edible or poisonous. Although the survey was focused on discovering heuristics or simple rules for foraging, many responses report using a more complex identification process. This process can, for example, involve looking up a mushroom 
in a definitive foraging guidebook and identifying them. It is therefore not a heuristic as it is a full and deterministic identification process. This is, though, a time-consuming process and it may not be feasible for a forager to enact such identification procedures for every mushroom encountered. Although the survey did not specifically ask respondents to describe such more complex procedures (see discussion on research design limitations below), many reported strategies resemble this identification process, such as:

- Learn to identify (the most common) poisonous mushrooms and lookalikes. (Table 4 .)

- Identify the mushroom in its natural terrain, and/or ensure it is not close to poisonous mushrooms. (Table 4.)

- Identify the mushroom once more at home, or when cleaning or preparing the mushroom. (Table 4.)

- Pick, identify and maintain whole mushrooms without breaking them (until preparation). (Table 4.)

- When identifying a mushroom, consult multiple information sources, including other people, books and online communities. (Table 4.)

It should also be noted that many respondents $(n=25)$ expressed their skepticism regarding the use of heuristics alone for identifying an edible mushroom, reporting the following foraging strategy:

- No heuristics suffice for identifying an edible mushroom (or distinguishing an edible from a poisonous one). (Table 5.)

- Some elaborations on this included the following responses: "Such rules of thumb do not exist, and one should learn to identify mushrooms by the species. Of course, white milk helps to identify a milk-cap as a milk-cap, and the web of a webcap as a webcap, and so on. But I would say they are pieces of the recognition puzzle, parts of identification wholes that help one towards the right evaluation, not rules of thumb."

- "There are no rules of thumb to distinguish a poisonous mushroom from an edible one if they are very much alike."

- "Never trust a single cue."

These are similar to typical pieces of advice in mushroom foraging guides, e.g.: "Never grasp on one identification cue when defining a species, but view the mushroom as a whole" (Korhonen, 2015, p. 12, translated by author). In other words, although simple rules of thumb or heuristics can be efficiently utilized in the identification process, particularly when ruling out certain subsets of mushrooms, they generally do not suffice alone to make inferences about edibility.

\subsection{Discussion}

"But what about poisonous and dangerous mushrooms! Not a word has been spoken of them," said the guest. "We do not worry about them," came the answer, "but leave all mushrooms to themselves, which we cannot recognize. Only this way can we be certain of them."

Quote from the first Finnish mushroom identification guide (Hisinger, 1863; translated by author).

The results provide evidence for the hypothesis that mushroom foragers use heuristics when identifying, making edibility-judgments of, and searching for mushrooms. $77 \%$ of foragers report that they often use rules of thumb when foraging. Moreover, some of the most common reported instances are heuristics that could classify as "simple" or "fast and frugal", such as "only pick the mushrooms you recognize" or "avoid white mushrooms altogether," whilst others utilized one-reason judgments to make inferences regarding edibility (e.g., the "white milk" of milk-caps of genus Lactarius).

However, a caveat is that some of the reported one-reason heuristics presuppose other more complex identification processes, including pattern recognition. For instance, distinguishing an edible milk-cap was reported by many to be possible by utilizing a single cue (bleeds white milk when cut, see Figure 7), but using this heuristic presupposes the more complex pattern-recognition task of recognizing mushrooms that belong to the (not especially distinctive) genus of milk-caps (Lactarius). This is also an interesting finding for the theory of ecological rationality, since it illustrates how fast and frugal heuristics can be used in tandem with more complex, possibly higher order, cognitive processes. For instance, one respondent answers:

- "If a mushroom bleeds milk it MIGHT be edible. Even then it must first be identified."

Accordingly, the typology developed in the Results section emphasizes that simple heuristics can be complemented by meta-heuristics (general principles for search and precaution), intuitive pattern recognition and complex and full identification.

Another core finding is the purpose which the most common heuristics seem to serve. Note that other than the very common "bleeds white milk" heuristic and the common "boletes have spongy pores (and are usually not poisonous)" heuristics (Table 5), the most common rules of thumb do not pertain to identifying an edible mushroom, but rather to ruling out subsets of possibly poisonous mushrooms, such white mushrooms (due to risk of confusion with A. virosa, see Figure 3). In other words, foragers seem to use "elimination-by-aspects" heuristics (Tversky, 1972), eliminating from consideration mushrooms with specific traits.

This can be understood as a process of uncertainty reduction or risk aversion - a precautionary heuristic that establishes clear boundary rules (Eisenhardt \& Sull 2001; Sull \& Eisenhardt, 2015) for the practice. By a priori ruling out those mushrooms that have a possibility of being deadly, 
and by only focusing on a limited amount of recognized 5 mushroom species, mushroom foragers are considerably reducing the amount of cognitive processing needed to make safe decisions. The "recognition" meta-heuristic ("pick only those mushrooms you recognize") is also an apparently old one and can be traced in written sources at least to the $1800 \mathrm{~s}$ (see epigraph of this section). To maintain the levels of uncertainty at a bearable level, many foragers $(n=38)$ also report a slow pace of learning new mushrooms.

Interestingly, precautionary heuristics have previously been suggested to be efficient foraging strategies in a simulation model. Bullock and Todd (1999, p. 533) found that precautionary foraging strategies are essential particularly in an environment with lethal mushrooms: "Since the consumption of a poisonous mushroom is fatal [...], every successful strategy there must proceed by rejecting subsets of mushrooms on the basis of cues which tend to make correct rejections." The survey data in the present study confirm that human foragers indeed use simple heuristics particularly to reject subsets of mushrooms. Three foragers describe these precautionary heuristics as follows:

- "All mushrooms are poisonous until proven otherwise."

- "I do not pick any white mushrooms."

- "If any uncertainty remains [after initial identification], the mushroom joins the alders [is thrown into the woods]."

The use of precautionary heuristics is a simple result of the fact that false positives are much worse than false negatives. Foragers thus find themselves in an asymmetrical (concave) payoff function (Taleb, 2012). For example, the benefits of eating a white mushroom are bounded (even deliciousness has its limits!), but potential costs are essentially infinite (death by poisoning). Thus, the precautionary heuristic of avoiding white mushrooms has culturally evolved for good reason.

Moreover, some heuristics may even be used without specific intention. Research in cultural evolution has documented in detail cases where inherited cultures "outsmart" individuals, also known as the "collective brain" phenomenon (Henrich, 2015; Muthukrishna \& Henrich, 2016). Curiously, the surveyed data also provided cases which suggest that some foragers use precautionary heuristics even though they were unaware why exactly they are doing so. For instance, one forager reports using the "avoid white mushrooms" heuristic without knowing why they do so:

- "I do not pick white mushrooms. I don't know why."

The responses also included many rather unique and complex mnemonics for foraging, such as the following:

5The "recognition" heuristic used by mushroom foragers (only pick those you recognize) is somewhat similar to the "recognition heuristic" studied in ecological rationality (Pachur et al., 2011). However, instead of using "recognition" as a positive cue for edibility (as the "recognition heuristic" would imply), mushroom foragers are using the recognition heuristic in the inverse: "non-recognition" is a cue for non-edibility, but recognition does not imply edibility.
- "On boletes: Bad girls have black fishnet stockings, and good girls have white stockings [author's note: the nonedible bitter bolete has a dark netlike pattern on its stalk, and the similar-looking porcini, a delicacy, has a whitish netlike pattern]. Nice aunty boletes wear an orange beret and brown, linty socks [a reference to the edible orange birch boletes]. Cortinarius caperatus is a Northern chap sitting by a bonfire: frost on his cap, an aurora-shaped scarf above his collar, and when young, a total prick [a reference to its phallic shape when young]."

Variable unique mnemonics like this could be expected from a practice such as mushroom foraging, which is inherited largely via vertical cultural transmission (from parents or grandparents) (Mesoudi, 2011, p. 60).

Research in ecological rationality emphasizes that decision-making is always context-specific, a product of the fitness between the mind and the environment. Indeed, foragers reported using several environmental cues as heuristics, leveraging statistical regularities in their environment to guide their search. These included associating specific mushroom species with particular trees or terrain types, as well as foraging in familiar patches or patches similar to previously bountiful ones. In the latter case, foragers may be utilising exemplars to guide their search. Moreover, the nature of ecologically rational decision-making implies that the used heuristics are applicable only in the environments where the foragers recurrently visit. Indeed, foragers often reported that the heuristic they use applies to their local forest. For instance, one respondent writes:

- "No poisonous boletes grow in my foraging patches, so all boletes can be picked except for those which have red straws. That one is the bitter bolete and is not edible."

Interestingly, cases are known where familiar heuristics used in unfamiliar contexts have led to fatalities. For instance, reports exist of tourists or foreigners using a heuristic associating whiteness with edibility when mushroom foraging in Finland (likely due to their familiarity with white champignons) - the exact opposite of one of the most common heuristics reported in this study, "avoid white mushrooms". This has led to several A. virosa poisonings (Hämeen Sanomat, 2018). Another example is the recent case of refugee mushroom poisonings in Germany (Connolly, 2015). Here, refugees had likely used familiar foraging rules from their Mediterranean home countries, where they had foraged for the bearded Amanita (Amanita ovoidea) and had mistakenly eaten the poisonous death cap (Amanita phalloides) in their new home in Germany.

Worth further discussion is also the meta-heuristic "If you find one mushroom (particularly funnel chanterelles and chanterelles), you are likely to find more of the same in the immediate vicinity" (Table 6). This is similar to a simple heuristic reported in a mushroom foraging study by Pacheco-Cobos et al. (2019), where the authors noted that foragers use the following area-restricted search heuristic to 
improve search efficacy: "search with slower and more tortuous movement after a recent encounter with a food item (intrapatch search) and move with more rapid and linear movement otherwise ("interpatch" search)". However, Finnish foragers mainly report using such a heuristic with mushroom species specifically known to grow in clusters (such as funnel chanterelles and chanterelles).

Finally, a caveat to interpreting the heuristics reported here through the lens of ecological rationality should be stated. Whereas the research tradition in ecological rationality and fast and frugal heuristics has traditionally focussed on categorization and decision-making rules through well-defined search processes (Berretty et al., 1999) or decision-making trees (Martignon et al., 2008), many of the heuristics reported by foragers in this study rather resemble simple, rough, and at times messy, guidelines or principles. They might therefore better be understood as "simple rules" as defined by Eisenhardt and Sull (2001; 2015; see also Marewski \& Hoffrage, in press, for contrasts between "simple rules" and "fast and frugal" heuristics).

The survey results give the impression of mushroom foraging as a highly intuitive practice. $90 \%$ of respondents report that they have a "strong hunch" of the mushrooms they will find prior to going on a foraging trip, and $76 \%$ report that they can anticipate what mushrooms grow in a patch based on a quick glance. Foraging also seems to be characterised by non-explicable "tacit knowledge" (Polanyi, 1969, 2009), as foragers report being capable of succeeding in their foraging tasks despite not being able to fully explicate their search or decision-making processes. These themes are returned to in Study 2.

A central limitation of the Study 1 is that, although the majority of respondents report using heuristics when foraging, the research design does not afford comparisons between the prevalence of simple or fast and frugal heuristics versus more complex decision-making processes. Uncovering how often exactly foragers use simple heuristics is therefore left for further research. Another limitation is the fact that the survey was conducted in an online environment. To more thoroughly study decision-making processes in foraging tasks, further field work and further ethnographic observations would be required to complement these findings.

\section{Study 2}

Study 2 studies how 894 foragers (the same as used in Study 1) use simple visual cues to guide their search for mushrooms, and how foraging experience refines this search process. Study 2 consists of the same participants and online survey design as Study 1.

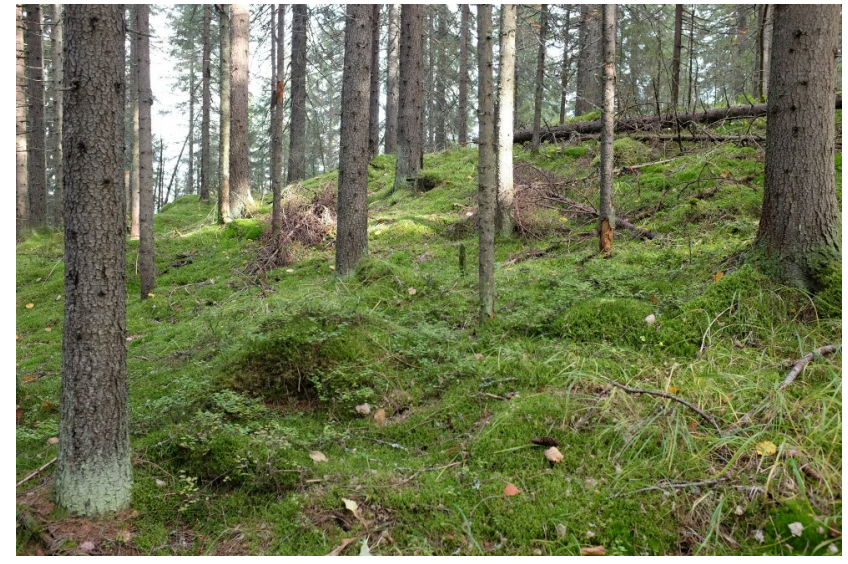

Figure 10: This picture of a coniferous forest includes some obvious cues for mushroom hunting: Spruces, fallen (decaying) trees, and a mossy and sloped landscape. Photograph by Janne I. Hukkinen with permission.

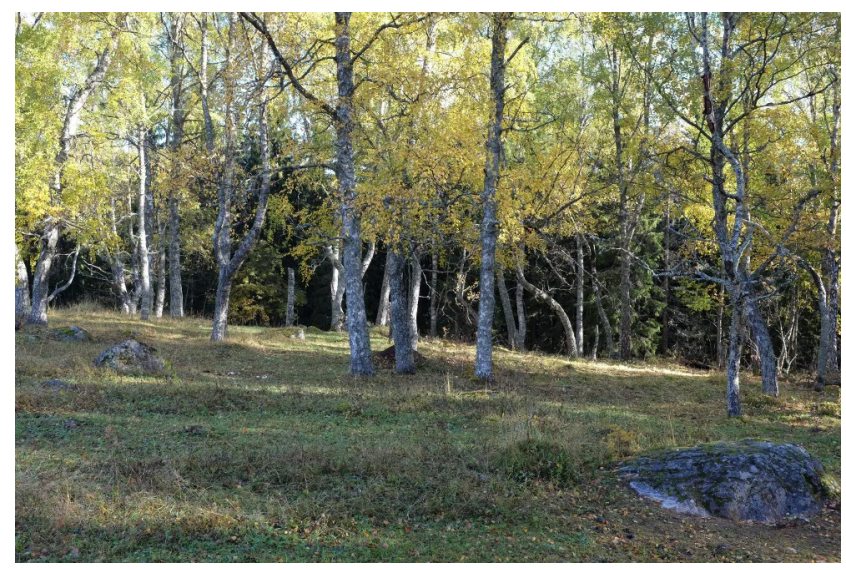

FIGURE 11: The cues for mushroom foraging in this birch forest include birches, grass, hay, light, dryness and a possibly pastural landscape. Photograph by Janne I. Hukkinen with permission.

\subsection{Method}

\subsubsection{Design}

Foragers were presented with a search task. Two pictures were presented in an online survey, one of a mossy coniferous forest (Figure 10), and another of a drier birch forest (Figure 11). Both pictures are from Finland, but the location and time of the photoshoot were not specified in the task.

Foragers were asked the question "What mushroom species would you search for in the terrain in this picture?", with an open response field. The aim of this task is to illustrate how little ecological information foragers can utilize to direct their search for mushrooms. Obvious ecological cues in Figure 10 include spruces, moss and a sloped terrain. Ecological cues in Figure 11 include birch trees, grass, 
hay, and a dry and illuminous, perhaps pastural or otherwise human-modified, landscape. Recall from Study 1 (Table 6 in particular) that many of these features are used as cues to guide search when foraging. The effect of foraging experience on the number of species mentioned is tested with a simple linear regression.

\subsubsection{Procedure}

To enable computational processing, the species or genera mentioned (nouns) were stemmed to their nominative singular case (since Finnish has fifteen noun cases). Other words were excluded from the dataset. Although the question asked for species, many answers were on the higher taxonomic rank of genus or family (e.g., boletes, milk-caps) or in folk taxonomy. These were chosen to be included in the analysis. In translation from Finnish to English, common names were preferred to binomial (Latin) names when available, since respondents rarely responded in scientific nomenclature.

\subsection{Results}

Two pictures were presented for the search task (Figures 10 and 11). The pictures were chosen to portray relatively little ecological information (Bruineberg et al., 2018; Gibson, 1979), but a sufficient number of cues to direct search. Overall, the respondents replied to the open question "What mushroom species would you search for in the terrain in this picture?" with little difficulty. A rich amount and diversity of answers were recorded.

Figures 12 and 13 are bar charts of the top 15 mushroom species or genera mentioned for each picture. Overall, Figure 10 proved to be a more promising and diverse foraging patch (3199 instances of mushrooms mentioned with 89 different species or genera) than Figure 11 (1656 and 79, respectively). The overall sentiment among the mushroom foragers was that the birch forest is a much inferior foraging patch to the mossy coniferous forest, e.g.: "I would not forage for mushrooms in this terrain, although I might find boletes. It somehow looks much too dry."

Experienced foragers reported a higher total number of mushroom species or genera (in the two pictures combined). A simple linear regression was calculated to predict the number of mushroom varieties mentioned based on self-reported experience $(\beta=0.08,95 \%$ CI $[0.06,0.10], \mathrm{p}=.000$, adjusted $\left.\mathrm{R}^{2}=0.075\right)$. This is illustrated in the raincloud plot (Allen et al., 2018) in Figure 14. The results suggest that more experienced foragers have more refined expectations of what mushrooms they expect to encounter in a presented terrain. This provides further evidence for the correlations found between variables Experience and Glance $(r=0.49)$ in the exploratory correlation plot of Figure 6. That is, experience predicts higher capacity to expect what to forage.

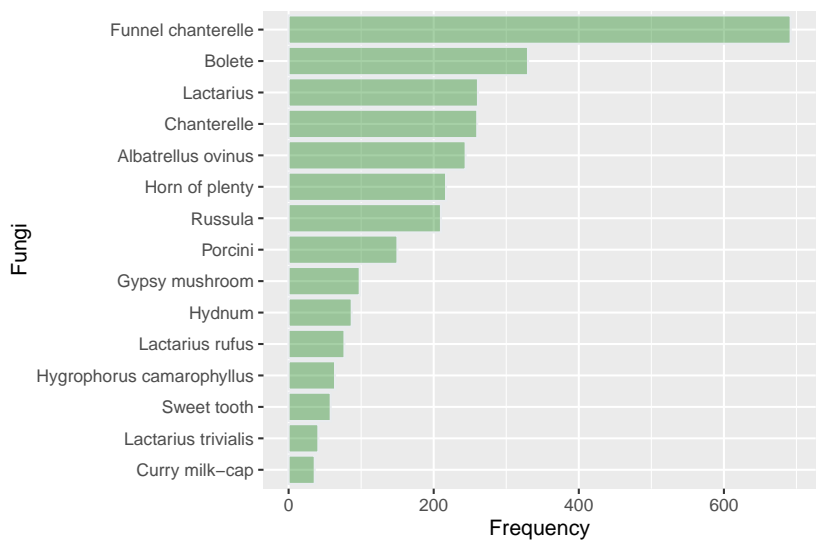

FIgure 12: Bar chart of the top 15 mushrooms identified in the search task for Figure 10 (coniferous forest).

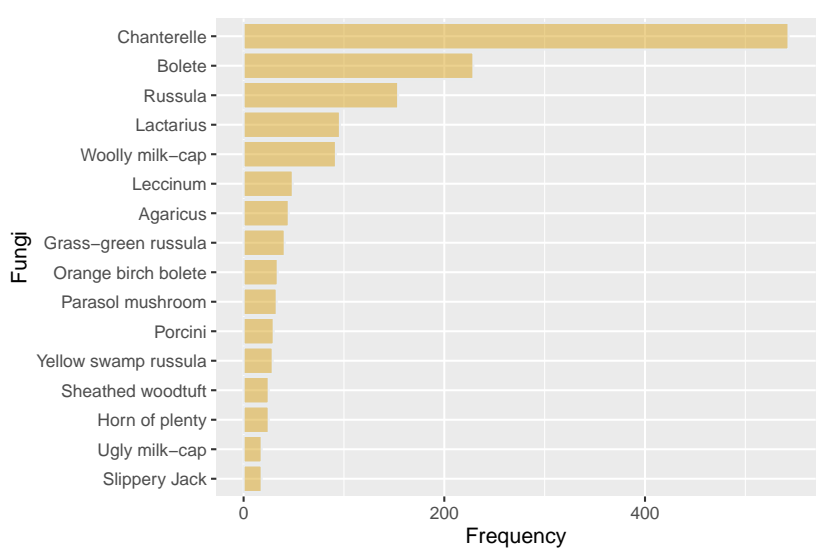

Figure 13: Bar chart of the top 15 mushrooms identified in the search task for Figure 11 (birch forest).

This might suggest that experienced foragers develop more refined selective attention (see Discussion below).

\subsection{Discussion}

This task illustrates how little ecological information foragers need to shape their expectations of catch as well as direct their search and attention. The results of Study 2 give some flesh to the previous finding (Figure 5, Study 1) that $76 \%$ of foragers agreed with the statement "With a quick glance of a given terrain, I know what mushrooms could grow in the area." The results of Study 2 also illustrate the use of many search heuristics (Table 6) in practice, such as associating specific mushrooms with specific plants or terrains. The mentioned species in Study 2 clearly differ between the two landscapes - e.g., the most common answer for Figure 10 (692 instances for funnel chanterelle) was mentioned only six times for Figure 11.

Despite the fact that, in Study 1, verbally explicating what makes a good foraging patch proved to be a difficult task for 


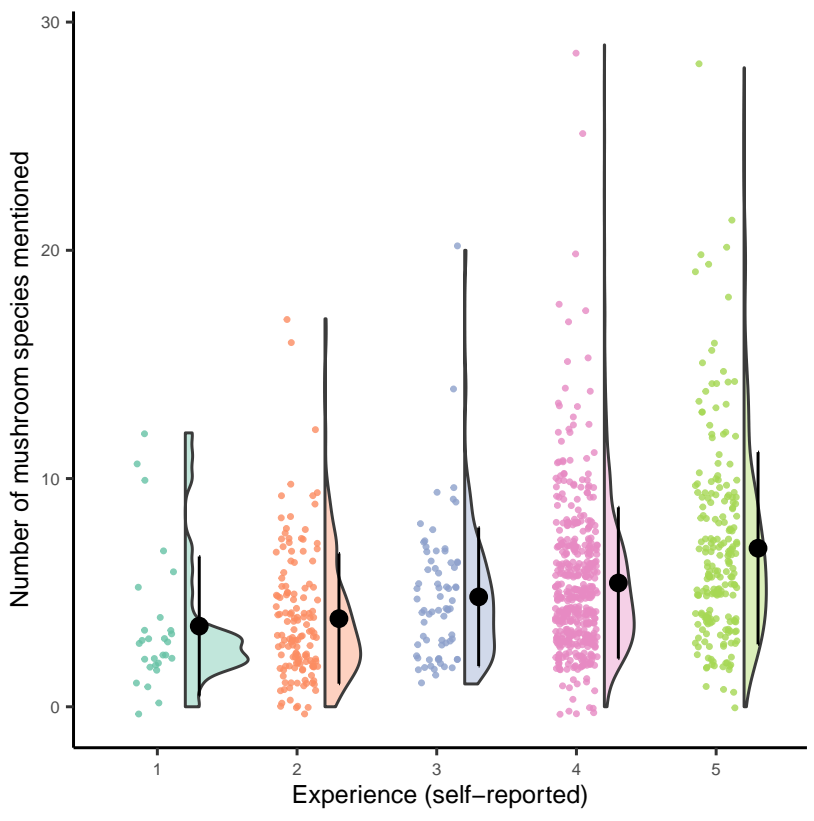

FIGURE 14: A raincloud plot of self-reported experience measured against the number of mushroom species or genera mentioned in Figs 10 and 11 (combined). The plot includes means (black points) with 95\% confidence intervals for each of the experience levels (measured on a low-to-high five-point scale), as well as all individual data points and density distributions.

foragers (a relatively low count of heuristics was reported in this domain), respondents had little trouble with identifying what grows where in the search task in Study 2. Furthermore, foragers seemed to be using at least some of the explicitly mentioned heuristics in Table 6. For instance, whilst only a total of 154 funnel chanterelle-related heuristics were mentioned in Study 1 (Table 6; e.g., that funnel chanterelles grow in mossy, sloped terrain with spruces), a total of 692 respondents mentioned they would search for funnel chanterelles in the terrain depicted in Figure 10, which portrays very little ecological information other than mossy terrain, spruces and sloped terrain. It might well be that mushroom foragers "know more than they can tell" (Polanyi 2009) in recognizing good foraging patches, as some foragers indeed explicitly stated. This tacit knowledge (Polanyi 2009; 1969) in foraging is particularly interesting, and it may involve the use of exemplars and pattern recognition in search procedures (Jones et al., 2000; Karlsson et al., 2008).

Moreover, Study 2 could inform further research in selective attention and perception. Although selective attention is by no means a new notion in psychology (James, 1892), to the authors knowledge it has not been studied extensively in human foragers. Selective attention has been better described in other animals (Kamil \& Bond, 2006). For instance, animals have been noted to develop "search images", or visual biases which selectively amplify certain features of the environment relative to others (Kamil \& Bond, 2006). Accordingly, one respondent notes on selective attention and the gradual development of a search image:

- "I have hunted mushrooms since I was a child, and my eye has been calibrated to mainly notice the few edible and beautiful or interesting mushrooms. Others I do not see."

Another forager describes developing a search image during the hunt:

- "After finding the first mushroom, you usually start finding more once your eye gets used to it."

Further studies on selective attention in mushroom foraging could prove to be a fruitful area of research in perceptual psychology, cognitive science and human behavioral ecology. A clear limitation of Study 2 is its online setting. Realworld perception is practically always accompanied by action (Gibson, 1979), and presenting a mere virtual 2D stimulus is likely insufficient to establish reliable results. Therefore, further research should study how foragers use simple visual cues to guide their search in natural settings and field experiments.

\section{General Discussion}

This article surveyed 894 Finnish mushroom foragers on their foraging strategies and heuristics. Most mushroom foragers (77\%), experienced foragers and novices alike, make common use of heuristics (rules of thumb), often resorting to "fast and frugal" one-reason decision-making when foraging. The present article gives ample evidence of mushroom foragers using simple or fast and frugal heuristics in decision-making and illustrates how foragers use ecologically valid environmental cues to guide their search.

Simple heuristics are particularly used to eliminate subsets of unknown and potentially poisonous mushrooms from consideration, e.g., by avoiding unrecognized or white mushrooms. The results illustrate how heuristics can play an important role in the cultural evolution of safe foraging practices, particularly by bounding the amount of uncertainty foragers deal with in decision-making. Yet whilst heuristics for identifying edible mushrooms are common, it is dubious whether simple heuristics alone suffice for judgments of edibility, since even the use of simple heuristics is often preceded by more complex cognitive processes, such as identifying the genus or family of the mushroom.

The results of Study 1 suggest a typology for categorizing decision-making in foraging. Foragers not only (1) use heuristics for identification and search, but also utilize (2) meta-heuristics, or rough guidelines, principles and precautionary measures to guide safe foraging practices, (3) intuitive pattern recognition and (4) complex and slow full identification processes. These four processes can be complementary. Study 2, in turn, illustrates how foragers can 
use simple visual cues, such as the presence of specific tree species, as heuristics to guide their search. Study 2 also suggests that foragers may develop selective attention through gain of experience.

Overall, the present study points many avenues for future research. For studies on decision-making and perception, it presents opportunities for further research in how heuristics are used in the often messy real-world, and how heuristics can be efficiently complemented by more complex decisionmaking processes. Looking ahead, it invites the reader to consider how traditional forms of uncertainty management might be applicable in our modern, technological, and riskcalculative world. For instance, with the surge of AI and mobile (computer vision) applications for identifying plants and mushrooms, it is reasonable to ask whether these new applications deal with uncertainty as robustly as culturally evolved traditional heuristics do. Looking to history, the results invite us to consider possible means by which other traditional or historical hunter-gatherer groups might have used simple or fast and frugal heuristics to facilitate safe foraging, and also point toward further research in how foraging societies and practices evolve culturally. These possibilities could be particularly relevant for research in cultural evolution, evolutionary psychology, and archaeology.

\section{References}

Allen, M., Poggiali, D., Whitaker, K., Marshall, T. R., \& Kievit, R. (2018). Raincloud plots: a multi-platform tool for robust data visualization. PeerJ Preprints, 6(e27137v1).

Berretty, P. M., Todd, P. M., \& Martignon, L. (1999). Categorization by elimination: using few cues to choose. In Simple heuristics that make us smart (pp. 235-254). Oxford University Press.

Bruineberg, J., Chemero, A., \& Rietveld, E. (2018). General ecological information supports engagement with affordances for 'higher' cognition. Synthese, 196, 497-541.

Bullock, S., \& Todd, P. M. (1999). Made to measure: Ecological rationality in structured environments. Minds and Machines, 9(4), 497-541.

Cocq, C. (2013). From the Árran to the internet: Sami storytelling in digital environments. Oral Tradition, 28(1), 123-142

Connolly, K. (2015). Germany attributes spike in mushroom poisonings to foraging refugees. The Guardian. (link)

Eisenhardt, K. M., \& Sull, D. N. (2001). Strategy as simple rules. Harvard Business Review, 79(1), 106-119.

Elävä perintö. (2019). Sienestys-wiki, Elävä perintö. https://wiki.aineetonkulttuuriperinto.fi/wiki/Sienestys

Elo, S., \& Kyngäs, H. (2008). The qualitative content analysis process. Journal of Advanced Nursing, 62(1), 107-115.
Encyclopedia Britannica. (2019). Fungus - Mycorrhiza. Encyclopedia Britannica. https://www.britannica.com/ science/fungus

Gibson, J. J. (1979). The ecological approach to visual perception. Houghton Mifflin.

Gigerenzer, G., \& Todd, P. M. (1999). Fast and frugal heuristics: the adaptive toolbox. In Simple heuristics that make us smart (pp. 3-34). Oxford University Press.

Hämeen Sanomat. (2018). Ulkomailta Suomeen muuttaneet eivät osaa varoa valkoisia sieniä - Myrkkysienen syöminen voi johtaa vakavaan maksavaurioon. Hämeen Sanomat. (link)

Henrich, J. (2015). The secret of our success: how culture is driving human evolution, domesticating our species, and making us smarter. Princeton University Press.

Hisinger, E. (1863). Sieni-kirja eli Sieni-Kallen oswiitta tuntemaan ja käyttämään syötäwiä sieniä. Suomen talousseura. https://helda.helsinki.fi/handle/1975/161

Jones, S., Juslin, P., Olsson, H., \& Winman, A. (2000). Algorithm, heuristic or exemplar: process and representation in multiple-cue judgment. Proceedings of the TwentySecond Annual Conference of the Cognitive Science Society, 244-249.

Kaaronen, R. O. (2019). The art of mushroom foraging: a phenomenological inquiry. In The side view journal (pp. 22-26). The Side View Press. https://thesideview.co/articles/the-art-ofmushroom-foraging/

Kamil, A. C., \& Bond, A. B. (2006). Selective attention, priming, and foraging behavior. Comparative cognition: experimental explorations of animal intelligence, 106-126.

Karlsson, L., Juslin, P., \& Olsson, H. (2008). Exemplarbased inference in multi-attribute decision making: contingent, not automatic, strategy shifts? Judgment and Decision Making, 3(3), 244-260.

Korhonen, J. (2015). Sieniopas: taskukirja sienten tunnistukseen. Readme.

Kozyreva, A., \& Hertwig, R. (2019). The interpretation of uncertainty in ecological rationality. Synthese, 196, 1-31.

Maaseudun tulevaisuus. (2017). Voit sienestää huoletta, jos tunnistat nämä kuusi tappavaa sientä. https: //www.maaseuduntulevaisuus.fi/ymparisto/voit-sienest \% C3\%A4\%C3\%A4-huoletta-jos-tunnistat-n\%C3\%A4m\% C3\%A4-kuusi-tappavaa-sient\%C3\%A4-1.205003.

Marewski, J. N., Gaissmaier, W., \& Gigerenzer, G. (2010). Good judgments do not require complex cognition. Cognitive Processing, 11(2), 103-121.

Marewski, J. N., \& Hoffrage, U. (In press). The winds of change: the Sioux, Silicon Valley, society, and simple heuristics. In Routledge handbook on bounded rationality.

Martignon, L., Katsikopoulos, K. V., \& Woike, J. K. (2008). Categorization with limited resources: A family of simple 
heuristics. Journal of Mathematical Psychology, 52(6), 352-361.

Mazzocchi, F. (2006). Western science and traditional knowledge. EMBO Reports, 7(5), 463-466.

Mesoudi, A. (2011). Cultural evolution. University of Chicago Press.

Metsäntutkimuslaitos. (2010). Luonnon virkistyskäyttö - Ulkoilutilastot 2010. http://www.metla.fi/metinfo/ monikaytto/lvvi/tilastot_2010/2010-taulukko-2-13.htm.

Ministry of the Environment. (2019). $E v$ -
eryman's rights. https://www.ymparisto.fi/enUS/Nature/Everymans_rights(27721)

Muthukrishna, M., \& Henrich, J. (2016). Innovation in the collective brain. Philosophical Transactions of the Royal Society B: Biological Sciences, 371(20150192).

Norman, G. R., \& Brooks, L. R. (1997). The non-analytical basis of clinical reasoning. Advances in Health Sciences Education, 2(2), 173-184.

Norman, G. R., Young, M., \& Brooks, L. R. (2007). Nonanalytical models of clinical reasoning: the role of experience. Medical Education, 41(12), 1140-1145.

Pacheco-Cobos, L., Rosetti, M., Cuatianquiz, C., \& Hudson, R. (2010). Sex differences in mushroom gathering: men expend more energy to obtain equivalent benefits. Evolution and Human Behavior, 31(4), 289-297.

Pacheco-Cobos, L., Winterhalder, B., Cuatianquiz-Lima, C., Rosetti, M. F., Hudson, R., \& Ross, C. T. (2019). Nahua mushroom gatherers use area-restricted search strategies that conform to marginal value theorem predictions. Proceedings of the National Academy of Sciences, 116(21), 10339-10347.
Pachur, T., Todd, P. M., Gigerenzer, G., Schooler, L., \& Goldstein, D. G. (2011). The recognition heuristic: a review of theory and tests. Frontiers in Psychology, 2.

Polanyi, M. (1969). Knowing and being: essays. University of Chicago Press.

Polanyi, M. (2009). The tacit dimension. University of Chicago Press.

Simon, H. A. (2000). Bounded rationality in social science: today and tomorrow. Mind \& Society, 1, 25-39.

Sull, D. N., \& Eisenhardt, K. M. (2015). Simple rules: how to thrive in a complex world. Houghton Mifflin Harcourt.

Taleb, N. N. (2012). Antifragile: things that gain from disorder. Random House.

Tilastokeskus. (2019). Keskivertosuomalainen. http: ]/tilastokeskus.fi/keskivertosuomalainen.html,

Todd, P. M., \& Brighton, H. (2016). Building the theory of ecological rationality. Minds and Machines, 26(1), 9-30.

Todd, P. M., \& Gigerenzer, G. (2007). Environments that make us smart: ecological rationality. Current Directions in Psychological Science, 16(3), 167-171.

Todd, P. M., \& Gigerenzer, G. (2012). Ecological rationality: intelligence in the world. OUP USA.

Tversky, A. (1972). Elimination by aspects: a theory of choice. Psychological Review, 79(4), 281-299. 